\title{
The Impact of Expertise on the Capture of Sketched Intentions: Perspectives for Remote Cooperative Design
}

\author{
Jennifer Sutera ${ }^{1}$, Maria C. Yang ${ }^{2}$, Catherine Elsen ${ }^{1}$ \\ ${ }^{1}$ LUCID-ULg, University of Liège, Belgium \\ jsutera@alumni.ulg.ac.be; catherine.elsen@ulg.ac.be \\ ${ }^{2}$ Department of Mechanical Engineering and Engineering Systems Division, MIT, \\ Cambridge, USA \\ mcyang@mit.edu
}

\begin{abstract}
The paper describes the way expertise and field-knowledge can impact the transfer of graphical intentions during architectural cooperative design. The analysis of 28 controlled experiments reveals what matters in transmitting architectural intents and more specifically underlines how novices' intuitive, deductive processes based on previous and embodied experiences interestingly complement experts' knowledge of the architectural field and its semantics. The results directly inform how we, as researchers, designers and engineers, should take advantage of both novices' and experts' strategies to develop tools, methods or interfaces to support next generation cooperative design.
\end{abstract}

Keywords: Cooperative design in architecture; transfer of design intents; expertise.

\section{Introduction}

Cooperative situations are nowadays the norm in almost any design field and architecture is no exception. At any design stage of an architectural project, stakeholders from different backgrounds and levels of expertise are frequently seen working together in dynamic environments, and remotely from each other. Among the numerous issues stakeholders face in such working environments, preserving efficient and effective communication certainly remains a decisive goal: anyone involved in transmitting an intention wants to be correctly understood.

The chosen medium for communication to support this transmission depends on the field, the type of collaboration, the stakeholders involved and the type of project. In the field of architecture, this communication traditionally occurs through graphical exchanges, naturally augmented with conversations and gestures. More specifically during the early stages of a design project, free hand sketching remains one of the preferred tools for architects because of its intuitive, natural and efficient characteristics $[1 ; 2]$. The use of digital tools to support remote collaborative design is also spreading, from emailing CAD representations to immersive environments that re-create a virtual sense of co-presence [3]. In the field of Sketch-Based Interfaces, researchers also look into free-hand sketching's natural and intuitive qualities to develop tools to support graphical remote cooperation [4].

Through our research, we want to help those researchers better understand how designers share information and intentions, in order to make these next generation tools, interfaces and methodologies more efficient and more reflective of real working habits. 


\section{State of the Art}

In vernacular societies, where human-made objects were conceived, made, and used by the same person [5], the design process could be theoretically estimated as the result of a single actor. The industrial revolution, however, confirmed the necessity of tasks' repartition between several stakeholders, among them the designer (or engineer - the person who conceives an object), the maker (the person who produces it), the consumer (the person who orders it) and the user (the person who experiences it). Today, putting aside the fact that design projects constantly and dynamically grow in size and in complexity, one also has to deal with the geographical distance separating (for instance) teams of architects or engineers from building or production sites.

These distant situations require adapted, augmented solutions for efficient remote cooperative design. In order to gain desirability and quick adoption, those next-generation interfaces, tools and methods should be innovative while respectful of stakeholders' practices and needs. As Norman and Verganti adequately summarize: "radical innovation driven by meaning change can be design driven through better understanding of potential patterns of meanings" [6]. In the case of cooperative design, one should consequently take a leap forward and project new ways to work and design together distantly, while always preserving the essential, qualitative aspects of cooperation.

In that regard, Baker, Détienne and Burkhardt researched the key indicators to assess the quality of collaboration (seen as a process rather than simply an outcome). Among the seven highlighted keys, two are of particular interest for this paper: to sustain first some mutual understanding and, second, the information exchanges during problem solving [7]. We summarize here those two keys under the overall goal of efficiently transferring intentions.

Several aspects could endanger this transmission of intentions: the erosion of psychosocial relations, due to the absence of physical co-presence; the incompleteness and inadequacy of shared information, weakened by less straightforward knowledge-sharing models; the illegibility of the shared information, impacted by badly designed IT environments or by lacking common ground between stakeholders of various backgrounds. In the field of distant architectural design, and more specifically during early stages of the design process, efficiency of remotely shared external representations is another key aspect underlying this intricate process of transferring intentions [8].

The role graphical representations play in supporting remote collaborative architectural design has already been extensively studied [9]. Some of our previous work in product design has specifically investigated how designers deal with foreign external representations, underlying the graphic strokes essential to the faithful transmission of an intention [10]. This current research rather focuses on architectural design, and more specifically on 2D plan representations, supposedly more straightforward in transferring and understanding intentions given the limited set of (quasi) universal architectural symbols and codes used in this field [11]. This paper questions whether this limited set of symbols and codified representations could constitute an interesting safe-pass to efficient transmission of intentions, and how the wide diversity of stakeholders taking part to a building project (from the architectural creative process to the building site) and the discrepancy of their respective expertise might impact this transmission. 


\section{Approach}

To investigate how the level of expertise might impact the capture of architectural intentions, a series of controlled experiments ( 20 to $45^{\prime}$ in duration) was set up. Participants with diverse architectural backgrounds were asked to copy a reference sketch (provided in hardcopy, see Figure 1) representing a two-dimensional house plan, intentionally blurry and incomplete but presenting a layout familiar to a wide range of participants and composed by a limited set of traditional architectural symbols. Free hand sketching was favored here because of its popularity among trained architects and its ease-of-use given the low expertise of some participants in dealing with other design tools.

Participants were trained to the think aloud method [12] (through a simple Hanoi tower exercise) and then asked to verbally comment their re-copying process, with no additional requirements in terms of exactness, style or time-efficiency. Strokes and verbalizations were recorded through the Smartpen ${ }^{\mathrm{TM}}$ technology that allowed a sequential replay of the data.

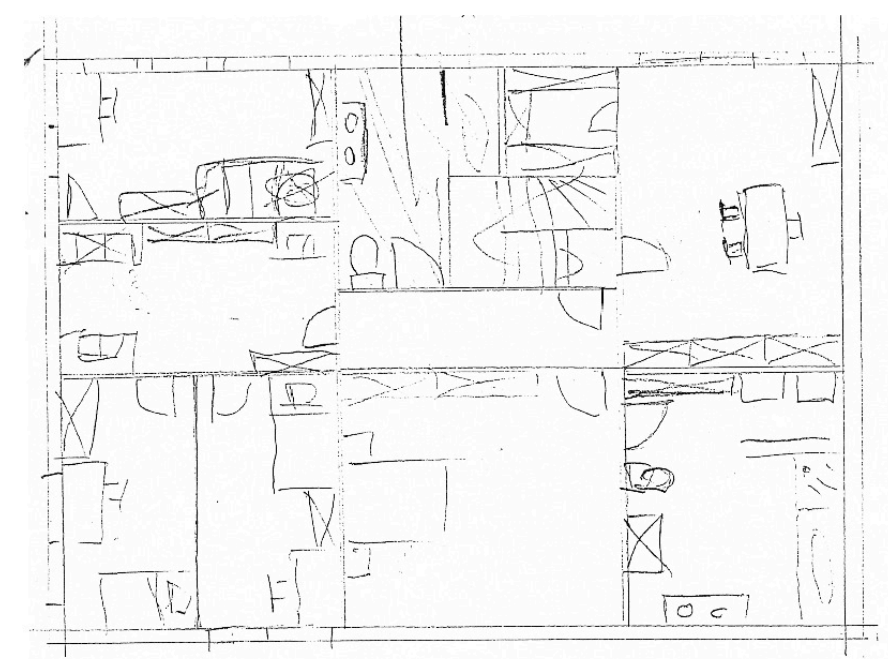

Fig. 1. The reference sketch of a two-dimensional, first-floor house plan.

To investigate the impact of expertise on capturing the sketched architectural intention, 28 participants (mainly graduate students) were selected based on their level of architectural knowledge and were classified following three categories:

- 10 participants with no specific knowledge in architecture (i.e., not following any class in architecture) were selected as "novices";

- 8 participants with a limited background in architecture (e.g. did not major in architecture but did take lessons or had significant contacts within this field) were considered as "familiar";

- 10 participants specifically studying architecture and close to finishing their degree were selected as "experts". 
Once all experiments completed, graphical elements of each copied sketch were coded in regard to the reference elements in the reference sketch. Graphic and verbal recorded data was analyzed quantitatively and qualitatively from different perspectives, such as the expertise of each participant, its interpretation and perception of the reference elements and the nature of the reference components.

\section{Results}

The first important result concerns the overall capture of the reference sketch. Interestingly, and in spite of its blurry and incomplete aspects, it appears that the reference sketch is globally accurately copied by all participants, regardless of the expertise level. Indeed, $43 \%$ of the reference elements (graphic units of the reference sketch, e.g. a door, a bed, a wall...) were copied by $100 \%$ of the participants, and $76 \%$ of those elements were even captured by 93\% of the participants. This confirms the (quasi) universal quality of 2D architectural plan representations: surpassing the blurry aspect of the reference sketch, even participants with no architectural expertise were able to recognize symbols that we all frequently encounter, for instance in real estate advertisements or fire-escape plans.

In previous research focusing on concurrent cognitive actions involved during design, Kavakli and Gero compared the cognitive processes of one novice and one expert architect during free-hand sketching and showed how the design thinking differed from one to the other. Their results revealed that the expert architect required three times less actions to reach the final version of his sketch; realized the design experiment three times faster than the novice and demonstrated an organized mind with strong focus on the task. The novice architect, on the other hand, did generate and investigate several options and aspects of the task simultaneously (therefore scattering his attention), but was also more inclined to more creative, innovative discoveries because of his potentiality to create much more unconventional associations [13].

Our results show a similar tendency this time in the process of capturing and interpreting a drawn intention. Figure 2 illustrates how experts extensively used their fieldknowledge when recopying the sketch $(44,7 \%$ of their actions being supported by a reflection nurtured by this architectural knowledge), while another $44,7 \%$ of actions was not orally commented (demonstrating the difficulty to verbalize highly usual, implicit actions). The remaining 10,6\% was performed using an indirect reflection, defined by Hattenauer as parallel strategies (association, intuition and similarity) people use when facing unknown situations [14]. Novices, on the other hand, used references to architectural knowledge in only $34 \%$ of the occurrences, relying more on indirect reflections $(38,1 \%$ of actions), nurtured by their personal way of experiencing space and previous experiences as an "inhabitant" of a house (for instance in terms of what furniture to expect inside a bedroom). 


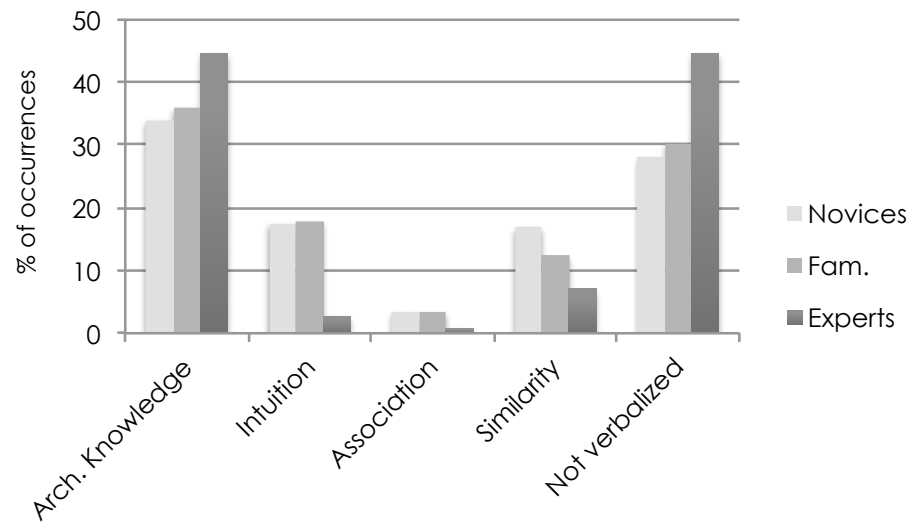

Fig. 2. Strategies used by different levels of expertise in the process of capturing a sketched intention.

Interestingly, our results additionally show how the three groups of participants differently dealt with the blurry and erroneous parts of the reference sketch. Figure 3 for instance shows how a 'door' element, originally badly drawn in the reference sketch (with a triangular shape instead of its standard "quarter-circle" representation, see picture extract on the right) is differently appropriated. It appears that while novices and familiar participants all recopy the item and correctly verbalize it as 'a door', most experts do not perceive it as such, some erroneously understanding it, others deciding not to copy it at all (perhaps considering it as a design mistake).

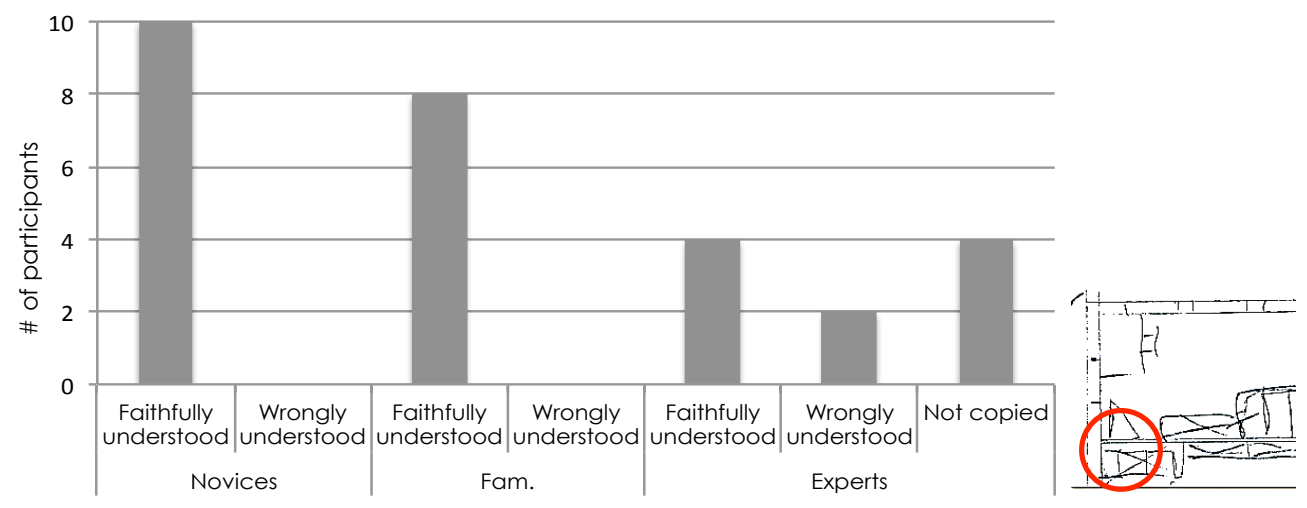

Fig. 3. Various understandings of a 'door' element.

We suggest that experts mainly base their recopying process on concepts and symbols that they have previously learned. They automatically react to the reference sketch by using their knowledge of architectural semantics, favoring efficiency and preferring not to spend too much time on what could not be immediately understood. Novices, on the other hand, are less trained to read these symbols and therefore approach them differently. Figure 4 illustrates two learning curves, showing how the number of key symbols (triggering the recognition process) increases with expertise, while conversely the number of confusing 
symbols (creating misunderstandings) diminishes with expertise. The remaining confusing elements for experts, as suggested by Figure 3, mainly relate to errors voluntarily left inside the reference sketch that they more hardly dealt with.

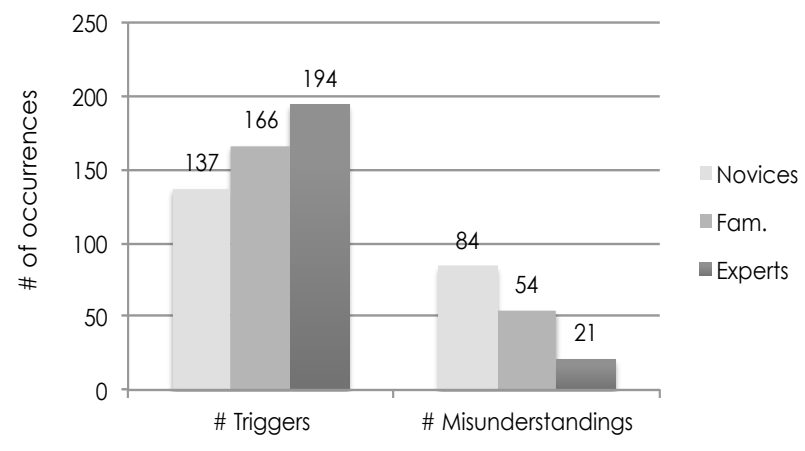

Fig. 4. Impact of expertise on perception of elements.

Besides being more disposed to discovery during design [13], the results of our study moreover suggest that the novice participants, with their flexibility, logical thinking and open-mindedness, better understand the most vague, unclear and erroneous parts of a sketch. They make more frequent reference to previous experiences, identifying parts of the blurry sketch thanks to what they encounter during everyday-life. They thus more easily identify symbols representing a real piece of furniture (for instance a toilet seat), clearly figuring a function (not too far from a kitchen should sit a dining table) or picturing a dynamic sensation (for instance, the dynamic movement of opening a door [15]).

Recurrent verbalizations moreover illustrate how participants expect some furniture to stand in each room. Bedrooms for instance are basically defined by the existence of a bed, sitting rooms by a couch, bathrooms by a toilet seat and offices by a desk and a chair. Similarly to the principal curves, found to constitute the graphic essence of shared representations in product design [10], standard symbols of pieces of furniture at first sight seem to play an essential, semantic role in the graphic transfer of an architectural intention. Once these essential components located, each group of participants nevertheless differently treated their respective symbols. Figure 5 shows differences related to strict faithfulness to the reference sketch (and its original intentions) : experts tend to slightly modify the symbols to implement their own drawing conventions (but still matching the overall codification), while novices are more respectful of the original drawing.

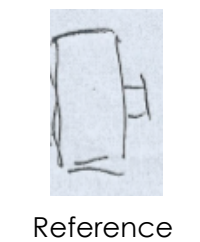

representation

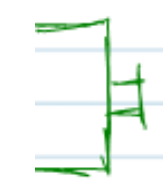

Novice representation
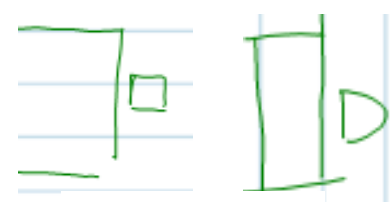

Expert representations

Fig. 5. Different representations of a desk, in regard of the level of expertise. 
All drawn symbols of pieces of furniture cannot yet be considered as essential to a robust transfer of intent, as illustrated by Figure 6 . Three representations of a closet (traditionally represented by a rectangle crossed by two diagonals) are displayed, respectively the reference, novice and expert representations. If the experts have no problem recognizing and copying the closet, novices experience more difficulties in apprehending the object since it is less intuitively assimilable to any lived experience. Most of the time not understanding its meaning, novices sketched something completely different from the reference sketch, therefore totally ignoring the standard semantic architectural codes. Interestingly, novices indeed faithfully copied elements especially when they were positive about their functions, and otherwise would not pay attention to any particular convention, the "mysterious objects" making no sense, requiring no particular attention and consequently being altered.

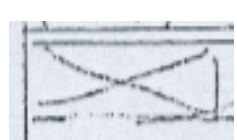

Reference representation

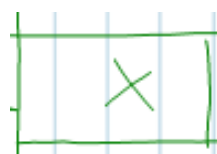

Novice representation

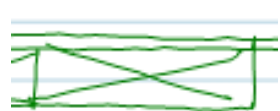

Expert representation

Fig. 6. Different representations of a closet, in regard of the level of expertise.

\section{Conclusion}

The results of this preliminary study suggest that architectural intentions carried out by free-hand sketches are globally well captured by all but that there are differences in understanding and appropriating those graphic intentions according to the level of architectural expertise.

Experts, given their high knowledge in architectural semantics, easily and quickly capture most of the intentions without investing a lot of time or attention. While misunderstanding fewer elements, they also grant less interest to more blurry and erroneous parts of the reference sketch and tend to more systematically apply their own drawing conventions.

Novices, on the other hand, more extensively rely on previous living and bodily experiences to understand the sketch and therefore more easily deal with the blurriest parts of the freehand sketch. They tend to more faithfully represent the initial intention, except when they encounter difficulties with symbols less intuitively understandable: in that case they don't hesitate to alter the representations and to depart from the architectural semantic language.

In the context of remote architectural cooperation, standardized symbols inside 2D architectural plans could have been prematurely considered as the unique and essential components of a graphic intention. Yet our results suggest caution when transferring such intentions between stakeholders with different backgrounds and expertise. Next generation cooperative support tools, methods and interfaces should keep at sight both novices' and experts' strategies and take inspiration from both intuitive, deductive novices' embodied experiences and field-knowledge experts' efficiency in order to insure optimal cooperation between stakeholders experiencing some geographical and background distance. 


\section{References}

1. Schön, D.A., Wiggins, G. (1992). Kinds of Seeing and their Functions in Designing. Design Studies, vol. 13, n², pp. 135-156.

2. Dogan, F. and Nersessian, N.J. (2010). Generic abstraction in design creativity: the case of Staatsgalerie by James Stirling. Design Studies, 31, pp. 207-236.

3. Dorta, T., Kalay, Y., Pérez, E. and Lesage, A.-M. (2011). Comparing immersion in remote and local collaborative ideation through sketches: a case study. CAAD Futures 2011 Proceedings.

4. Elsen, C., Demaret, J-N., Yang, M-C., and Leclercq, P. (2012). Sketch-Based Interfaces for Modeling and Users' Needs: Redefining Connections. AIEDAM Vol. 26, ${ }^{\circ} 3$.

5. Jones, J. C. (1970). Design methods: Seeds of human futures. Chichester: John Wiley.

6. Norman, D.A. and Verganti, R. (2012). Incremental and radical innovation: design research versus technology and meaning change. Submitted to Design Issues.

7. Baker, M., Détienne, F. and Burkhardt, J-M. (2013). Quality of collaboration in design: articulating multiple dimensions and viewpoints. 1st Interdisciplinary Innovation Conference, Telecom ParisTech.

8. Darses, F. (2009). Résolution collective des problèmes de conception. Le Travail Humain, 2009/1 (Vol. 72), pp. $43-59$.

9. Safin, S., Juchmes, R. and Leclercq, P. (2012). Use of graphical modality in a collaborative design distant setting. Work: A journal of prevention, assessment and rehabilitation, Supplement 1.

10. Elsen, C., Darses, F. and Leclercq, P. (2012). What do strokes teach us about collaborative design? Lecture Notes in Computer Sciences 7467, Springer, pp 114-125.

11. Dessy, J. (2002). De l'emploi des symboles dans les esquisses architecturales. Thesis, Université de Liège.

12. Van Someren, M., Barnard, Y., and Sandberg, J. (1994). The Think Aloud Method: A Practical Guide to Modelling Cognitive Processes. Academic Press, London.

13. Kavakli, M. and Gero, J.S. (2001). The Structure of Concurrent Cognitive Actions: a Case Study on Novice and Expert Designers. Design Studies, vol. 23, n 1, pp. 25-40.

14. Hattenhauer, D. (1984). The Rhetoric of Architecture: a Semiotic Approach. In Communication Quarterly, vol. 32, pp. 71-77.

15. Eco, U. (1980). Function and Sign: the Semiotics of Architecture. In: Signs, Symbols and Architecture, pp. 11-69. 